\title{
Aplicación de la Lógica Dominante del servicio (LDS) en el sector turístico: el marketing interno como antecedente de la cultura de co-creación de innovaciones con clientes y empleados
}

\author{
Service Dominant Logic in the tourism sector: \\ internal marketing as an antecedent of an \\ innovations' co-creation culture with clients and \\ first-line employees
}

\author{
Nuria García RodríGueZ ${ }^{1}$ \\ Begoña Álvarez Álvarez ${ }^{1}$ \\ M. ${ }^{a}$ Leticia Santos ViJande ${ }^{1}$ \\ Universidad de Oviedo (España)
}

Recibido el 24 de mayo de 2010 y aceptado el 29 de septiembre de 2010

$\mathrm{N}^{\mathrm{o}}$ de clasificación JEL: M12, M31

DOI: $10.5295 /$ cdg.100238ng

\section{Resumen:}

Este trabajo trata de profundizar en la comprensión del concepto de marketing interno (MI), considerado como un recurso operante desde la óptica de la Lógica Dominante del Servicio (LDS), así como en su influencia en la obtención de resultados empresariales superiores a los de la competencia. Para ello, se examina el efecto del MI en la predisposición de las empresas analizadas a que sus clientes y empleados de primera línea participen en el desarrollo de innovaciones de servicio, ampliando de este modo, de acuerdo con la LDS, las oportunidades de co-creación de valor disponibles para las organizaciones. Para contrastar las hipótesis planteadas se aplica un análisis de ecuaciones estructurales a la información facilitada por los gerentes de 240 hoteles de una muestra de ámbito nacional. Los resultados obtenidos permiten concluir que aquellas empresas hoteleras que aplican en mayor medida una estrategia de MI están más predispuestas a la co-creación de innovaciones con sus clientes y empleados de primera línea. Por su parte, estas subculturas favorecen la consecución de mejores resultados de las empresas hoteleras a largo plazo, en relación a sus competidores, tanto con sus clientes, como en indicadores financieros y de mercado.

\footnotetext{
${ }^{1}$ Universidad de Oviedo, Facultad de Economía y Empresas, Dpto.de Administración de Empresas, Avda. del Cristo, s/n33071- Oviedo.Email: nuriagr@uniovi.es; mbalvarez@uniovi.es; 1santos@uniovi.es

* Este trabajo ha sido posible gracias a la financiación proporcionada por el Ministerio de Educación y Ciencia, dentro de su Plan Nacional de I+D+i (2008-2011), al proyecto titulado "Estrategias proactivas y reactivas de recuperación del servicio en el sector turístico: Análisis del papel de la innovación guiada por el mercado como instrumento competitivo a largo plazo" (MICINN-ECO2008-03698/ECON).
} 


\title{
Palabras clave:
}

Lógica dominante del servicio, marketing interno, co-creación, resultados.

\begin{abstract}
:
This research seeks to achieve a deeper understanding of the Internal Marketing (IM) concept, considered as an operant resource, according to the Service Dominant Logic (SDL) theory, as well as to analyze the IM contribution to the achievement of competitive advantage. To this end, the research examines the IM effect on the organizations' predisposition to involve their clients and first-line employees in the development of new services, widening in this way the opportunities for value co-creation, again from a SDL viewpoint. The conceptual model is tested on a 240 national sample of hotels using structural equations modelling. The results support that those firms more involved in IM practices are also more willing to co-develop new services with their customers and first-line employees, which ultimately improve in a sustained way customer-related performance and overall firm's performance.
\end{abstract}

\section{Keywords:}

Service dominant logic, internal marketing, co-creation, performance. 


\section{INTRODUCCIÓN}

El sector turístico constituye uno de los principales motores generadores de crecimiento económico en España. En 2008, este sector supuso una contribución al PIB del 10,5\%, contabilizó 2.889.153 trabajadores (el 12,6\% de los activos de la economía nacional y el 19,7\% del sector servicios), y facturó más de 113.000 millones de euros (INE ${ }^{1}, 2009$ ). Por añadidura, España fue en este mismo año el primer país de la Unión Europea y el segundo del mundo que más ingresos registró por turismo, situándose en tercera posición dentro de los principales destinos a nivel mundial con 58 millones de visitantes (World Tourism Organization, 2009). El sector turístico incluye fundamentalmente actividades de restauración, ocio, transporte y alojamiento de los turistas. Dentro de las posibilidades de alojamiento, durante 2008 la oferta hotelera en España fue de casi 1,7 millones de plazas, siendo el segundo país de la Unión Europea en esta magnitud después de Italia. Los turistas alojados en hoteles efectuaron el 65,8\% del gasto total en alojamiento durante este periodo, en total 34.127 millones de euros, lo que supuso prácticamente un tercio de la facturación sectorial (INE, 2009). Todos estos datos ponen de manifiesto la importancia del sector turístico en la economía española junto con la especial relevancia de las empresas hoteleras dentro del sector.

Sin embargo, existen crecientes problemas de competitividad estructural en términos de su capacidad de generación de ingresos y efectos multiplicadores sobre otros sectores. Estudios recientes sobre el sector turístico en la Unión Europea (UE) advierten que las empresas turísticas de la UE están más impulsadas por el producto que por el consumidor y que el sector presenta una gran carencia de soluciones innovadoras para enfrentarse a retos mayores, como identificar nichos de mercado, reducir el carácter estacional de la demanda, mejorar las condiciones de trabajo, o incrementar la satisfacción y la lealtad de los clientes turísticos; lo que en su conjunto sugiere que se presta poca atención a la creación de valor añadido (Comisión UE, 2009).

En este sentido, desarrollos recientes del marketing, tales como la Lógica Dominante del Servicio (LDS) (Vargo y Lusch, 2004), pueden resultar de especial utilidad para guiar la gestión de las empresas de servicios turísticos hacia la creación de valor. Una de las premisas básicas sobre las que se asienta este enfoque establece que las empresas no entregan valor, sino que tan sólo hacen propuestas de valor dado que éste es creado por los clientes mediante el uso o consumo de los productos o servicios (Lusch et al., 2009). En este contexto, las empresas sólo pueden alcanzar un estado de co-creadoras de valor durante la interacción con sus clientes. Antes de la interacción, la posición de las organizaciones es la de facilitadoras de valor, puesto que tratan de proporcionar a sus clientes bienes y servicios que éstos puedan utilizar para obtener valor (Grönroos, 2008). No obstante, autores como Ballantyne et al. (2008) reconocen que las propuestas de valor pueden ser co-producidas por clientes y empresas, es decir, se puede colaborar en la co-creación de nuevos servicios o productos de tal forma que la empresa interactúa y negocia con sus clientes para desarrollar una propuesta de valor; este proceso, en sí mismo, se convierte en una parte de la co-creación de valor en la que puede participar la empresa (Figura 1).

\footnotetext{
${ }^{1}$ Datos relativos al año 2008 de la Cuenta Satélite del Turismo de España -CSTE-, que mide la evolución del PIB turístico y la contribución del turismo al conjunto de la economía española.
} 
En decir, es una forma con la que cuentan las empresas hoteleras para ampliar la creación de valor de sus clientes es precisamente permitirles colaborar en la co-creación de nuevos servicios turísticos.

Figura 1

\section{Co-creación de valor extendida mediante la co-producción}

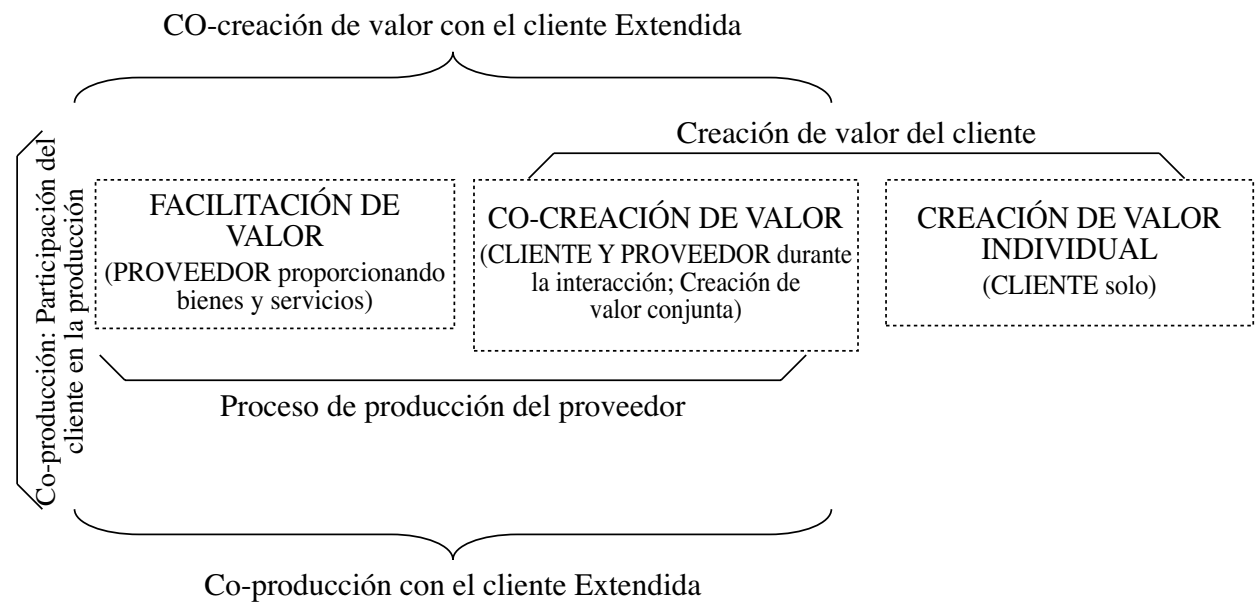

Fuente: Adaptado de Grönroos (2008)

No obstante, la co-creación con los clientes no es un proceso inmediato ni carente de obstáculos (Magnusson et al., 2003; Carbonell et al., 2009), por lo que es necesario saber cuáles son los factores que favorecen que las empresas turísticas aprecien la involucración de los clientes en la co-creación de nuevos servicios.

En este sentido, otra de las contribuciones clave de la LDS es la identificación de los recursos organizativos operantes, es decir, aquellos basados en conocimientos o habilidades, como la base de la ventaja competitiva o resultados superiores a los de la competencia (Madhavaram y Hunt, 2008). Entre estos recursos se encuentra el marketing interno (MI), el cual se puede concebir como una filosofía de gestión que promueve el desarrollo de estrategias y programas dirigidos a motivar, estimular, comprometer y favorecer el rendimiento de todos los empleados de la empresa (o mercado interno), lo que a su vez facilita la consecución de los objetivos organizativos con los clientes finales en el mercado externo (Gounaris, 2008; Domínguez et al., 2009).

Madhavaram y Hunt (2008) advierten de la necesidad de conocer con más detalle la influencia de los recursos operantes en las distintas actividades de la empresa, por lo que en este estudio se analiza el papel del MI como un posible antecedente de la valoración favorable de la co-creación de nuevos servicios con sus clientes en los hoteles. Así, entendemos que el MI crea el clima organizativo adecuado para incorporar la voz del cliente y apreciar su participación del desarrollo de innovaciones de servicio (Sharpley y Forster, 
2003; Nasution y Mavondo, 2008). El MI se selecciona como un recurso operante relevante para este estudio porque tiene una indudable repercusión en otra de las variables del modelo considerado: la valoración o apreciación de los empleados de primera línea como co-creadores de nuevos servicios. Así, el MI prioriza la estima y el bienestar del empleado mediante el diseño e implementación de prácticas de recursos humanos que satisfagan sus necesidades (Domínguez et al., 2009). De este modo, el MI constituye además una política especialmente importante para la gestión del personal en las empresas de servicios (Kelemen y Papasolomou-Doukakis, 2004), dado que los empleados son los encargados últimos de la provisión del servicio y condicionan de modo muy importante su nivel de calidad (Shiu y Yu, 2010), por lo que es imprescindible que se encuentren satisfechos en la organización.

El objetivo de esta investigación es analizar el grado en que las empresas hoteleras aplican el MI para gestionar sus recursos humanos y en qué medida este hecho puede influir en la valoración positiva de la co-creación de nuevos servicios con los clientes y con los empleados de primera línea (aquellos que están en contacto directo con el cliente). Es decir, se valora la influencia del MI en la predisposición cultural de las empresas hoteleras a que sus clientes y empleados participen en la co-creación de nuevos servicios. Además, se persigue estudiar la repercusión de la predisposición a co-crear en el desarrollo de innovaciones con ambos tipos de colectivos sobre los resultados que obtienen las empresas hoteleras con sus clientes y a nivel organizativo. La innovación en la empresa puede efectuarse en los propios productos o servicios comercializados, en sus procesos productivos, o en cualquier otro tipo de procesos organizativos que necesiten ser adaptados a la evolución del entorno (Santos y Álvarez, 2007). En este estudio, el tipo de innovación considerado es el referido exclusivamente al desarrollo de nuevos servicios, es decir, se investiga hasta qué punto los hoteles de la muestra están dispuestos a colaborar con sus clientes y empleados de primera línea en el diseño de nuevos servicios turísticos.

El trabajo se estructura como sigue. En primer lugar, se ofrece una revisión sobre la evolución teórica que ha seguido el concepto de MI en la literatura de marketing. En segundo lugar, se analiza cómo el desarrollo de una estrategia de MI incide en la valoración de la co-creación con los clientes y con los empleados de primera línea en el desarrollo de nuevos servicios en las empresas hoteleras. En tercer lugar, se discute la repercusión de ambos tipos de subculturas de co-creación, con empleados y clientes, en los resultados organizativos. En cuarto lugar, se presenta la metodología seguida para la realización del estudio empírico, y se exponen los resultados obtenidos de la validación de las escalas y de la estimación del modelo causal mediante el programa EQS. Finalmente se comentan las principales conclusiones teóricas e implicaciones para la gestión, así como las limitaciones del estudio y las líneas de investigación futuras.

\section{MARKETING INTERNO: CONCEPTO Y DIMENSIONES}

Las condiciones actuales de los mercados, tales como la creciente globalización y consiguiente aumento de la intensidad competitiva, o la cada vez mayor sofisticación de los deseos de los consumidores, provocan que muchas empresas de servicios persigan el logro de ventajas competitivas a través de la orientación al mercado y de una mejora permanente 
de sus servicios, con el objetivo de conseguir y mantener la satisfacción y la lealtad de sus clientes a largo plazo (Rowe y Barnes, 1998; Conduit y Mavondo, 2000). Sin embargo, la capacidad de la empresa para poner en práctica estos aspectos se ve en numerosas ocasiones condicionada por los empleados del servicio, lo que pone de manifiesto la necesidad de aplicar una estrategia de MI (Gounaris, 2008).

El significado y el contenido del MI no ha permanecido estático sino que ha sufrido una evolución a lo largo del tiempo. Dicha variación puede quedar resumida en las siguientes etapas (Rafiq y Ahmed, 2000):

Primera etapa: motivación y satisfacción del empleado. Los trabajos se centraron en cómo obtener empleados motivados y satisfechos de cara a mejorar la calidad de los servicios (Berry, 1981; Arndt, 1983). Partían de la idea de que para tener consumidores satisfechos, la empresa necesita también disponer de empleados satisfechos, dado que el personal juega un papel clave a la hora de proporcionar un servicio de calidad (Sasser y Arbeit, 1976). El medio fundamental para lograr empleados satisfechos consiste en tratarlos como si fueran verdaderos clientes, de modo que la atracción, retención y motivación del personal se convierten en actividades especialmente críticas.

Segunda etapa: orientación al consumidor. No es suficiente con que los empleados estén motivados para desempeñar mejor su trabajo, sino que además deben ser conscientes del consumidor, es decir, deben tener una mentalidad de ventas y estar orientados al consumidor (Grönroos, 1981; Barnes, 1989). Además, se considera al MI como un medio para integrar y coordinar las diferentes funciones vitales en las relaciones con los clientes en las empresas de servicios.

Tercera etapa: ejecución de la estrategia y gestión del cambio. Se considera al MI como una herramienta o recurso para ayudar a la empresa a que ejecute de forma efectiva su estrategia (Winter, 1985; George, 1990). En este sentido, el MI adquiere el rango de filosofía empresarial (Lings, 2004; Domínguez et al., 2009) centrada en la satisfacción del empleado y que promueve el desarrollo de los mecanismos necesarios dentro de la organización para conseguir la integración funcional, disminuir el aislamiento interdepartamental, reducir las fricciones internas y superar la resistencia al cambio (Rafiq y Ahmed, 1993). Se cree que el MI puede ser de aplicación en cualquier tipo de empresa y de empleados, no únicamente al personal del contacto con el cliente ya que se trata de una estrategia que persigue la creación de sistemas de trabajo de alto rendimiento para crear y lograr mayores competencias individuales y organizativas y, en última instancia, influenciar el rendimiento del negocio (Ahmed et al., 2003).

Los últimos trabajos centrados en la medición operativa del MI (Lings, 2004; Lings y Greenley, 2005; Gounaris, 2006; Tortosa-Edo et al., 2010) utilizan un constructo análogo en cuanto a sus dimensiones a la orientación al mercado operativa de Kohli y Jaworski (1990), pero referido a las prácticas de IM: generación de información sobre el mercado interno, diseminación de la información interna y respuesta a la información interna. Esta propuesta de tres dimensiones ha sido utilizada por Sanzo et al. (2007) como punto de partida para diseñar una escala operativa que permitiera medir el grado de utilización de las prácticas de MI por parte de las empresas de servicios. En el presente trabajo adoptamos dicha escala adaptada al ámbito de las empresas hoteleras. 


\section{MARKETING INTERNO Y VALORACIÓN DE LA IMPLICACIÓN DE CLIENTES Y EMPLEADOS EN EL DESARROLLO DE NUEVOS SERVICIOS}

Desde una perspectiva de marketing, para una empresa resulta esencial el conocimiento de los deseos de los consumidores para ofrecerles un servicio adaptado a sus necesidades, aumentando, en consecuencia, las posibilidades de éxito. Para lograr la adaptación del servicio a las exigencias de los clientes, los cuales, en la práctica participan activamente en el proceso final de producción y consumo del bien, la implicación de los mismos en el desarrollo de los nuevos servicios resulta también un factor clave ${ }^{2}$.

Existen evidencias sobre la interrelación que existe entre la orientación al mercado y el desarrollo del MI en la organización. El MI es considerado un antecedente del marketing externo (Bansal et al., 2001; San Martín, 2005) ya que el papel de los empleados resulta vital para conseguir satisfacer a los consumidores, y la posibilidad de que los empleados se vinculen con un proyecto de gestión orientada al mercado pasará inexorablemente por la existencia de un MI apropiado en la organización. Así, trabajos como el de Conduit y Mavondo (2000) contrastan empíricamente cómo la orientación interna de una empresa hacia sus empleados facilita el desarrollo de una orientación externa o al mercado. Por tanto, las empresas han de desarrollar estrategias adecuadas de MI de modo que sus empleados se encuentren satisfechos, dado que su satisfacción repercutirá en su buen hacer en la empresa y en las relaciones con los clientes (O’Reilly y Pfeffer, 2000).

A través del MI los empleados estarán concienciados de la importancia del consumidor y del papel que éste juega, por lo que tendrán interés en conocer sus deseos y necesidades para poder satisfacerlos (Counduit y Mavondo, 2000; Sharpley y Forster, 2003). En este contexto, entendemos que debería existir una disposición más favorable hacia la participación activa e implicación de los clientes en el desarrollo de nuevos servicios, la cual se canaliza mediante la colaboración con los empleados de la empresa (Nasution y Mavondo, 2008). Es decir, si el objetivo es la satisfacción del consumidor, la aplicación de un MI contribuirá favorablemente a la valoración de la implicación del cliente en el desarrollo de la innovación como consecuencia de la existencia en todos los empleados de una clara orientación externa. Sobre este último aspecto no existen videncias empíricas, por lo que ésta constituye una de las cuestiones que se pretenden contrastar a través de la presente investigación.

Como consecuencia, se propone la siguiente hipótesis de trabajo:

H1: La aplicación de un enfoque o estrategia de MI en las empresas hoteleras favorece la valoración de la participación de los clientes en el desarrollo de innovaciones.

En el contexto del marketing de servicios, la naturaleza intangible del producto convierte a los empleados en uno de los parámetros cruciales en el proceso de generación de valor del servicio de la organización. Los empleados de primera línea interactúan con los clientes, por lo que necesitan entender sus necesidades y adaptar el servicio de la empresa a las mismas. Adicionalmente, obtienen información sobre la competencia, ayudan a la entidad a comprender las necesidades exactas de los clientes y el mejor modo de satisfacerlas y promueven la imagen de la compañía (Gounaris, 2008).

\footnotetext{
${ }^{2}$ La implicación del cliente en la innovación de servicios se refiere al grado en el que las empresas colaboran con los clientes (actuales y/o potenciales) durante las distintas etapas del proceso de desarrollo de nuevos servicios (Alam, 2002, 2005).
} 
El MI incluye, entre otras, acciones tales como la selección, formación e incentivo de los empleados, la socialización de los trabajadores, el establecimiento de información abierta y precisa entre los empleados y la dirección, el empowerment y la participación en la toma de decisiones (Bansal et al., 2001; Naudé et al., 2003).

En concreto, esta última, la gestión participativa, equilibra la implicación de la dirección y sus subordinados en el procesamiento de la información, la toma de decisiones, o la resolución de problemas (Wagner, 1994). Una forma de implicar a los empleados en la organización es la consideración de éstos, por parte de la dirección, como una fuente importante de ideas en el desarrollo de nuevos servicios. En las organizaciones de servicios los empleados de primera línea constituyen una fuente de información esencial para guiar el desarrollo de innovaciones y tienen un papel determinante en su difusión y resultados (Santos et al., 2009).

En este sentido, aunque no existen evidencias empíricas al respecto, la valoración por parte de la empresa de la involucración de los empleados de primera línea en el desarrollo de nuevos servicios parece surgir como una consecuencia lógica de la aplicación de una estrategia de MI en la misma. Las empresas que realizan una gestión de personal bajo la óptica del MI manifiestan una especial preocupación por conocer las necesidades y habilidades específicas de sus empleados, establecer programas de formación y sistemas de incentivos y mejorar las comunicaciones verticales entre gerentes y los trabajadores, en aras a una mejora de la motivación y la satisfacción de los trabajadores (Vasconcelos, 2008; Domínguez et al., 2009; Rodríguez et al., 2009). Este énfasis en el reconocimiento de la importancia de los empleados y del trabajo que desarrollan en la organización puede llevar implícita la consideración por parte de la empresa de que las aportaciones que éstos pueden realizar en las iniciativas innovadoras que se lleven a cabo son especialmente relevantes y valiosas (Gwinner et al., 2005). Al fin y al cabo, en las empresas de servicios, en las que los empleados de primera línea son la cara visible de la organización e interactúan directa y estrechamente con los clientes, éstos desempeñan un papel crucial en la captación de las exigencias del mercado, disponiendo de un conocimiento muy valioso para el desarrollo de nuevos servicios en la empresa.

En este sentido, se plantea como segunda hipótesis de la investigación:

H2: La aplicación del MI en las empresas hoteleras favorece la valoración de la participación de los empleados de primera línea en el desarrollo de innovaciones.

\section{EFECTOS DE LA CULTURA DE CO-CREACIÓN EN LOS RESULTADOS}

La premisa básica que subyace en la propuesta de implicar a los consumidores y empleados de primera línea en la creación de nuevos servicios, haciéndoles tomar parte activa en el proceso de desarrollo de los mismos, es que la co-creación provoca efectos positivos en los resultados empresariales (Vargo y Lusch, 2004; Ballantyne et al., 2008). En este estudio se analiza el efecto de la cultura de co-creación en dos tipos de resultados, primero y como variable mediadora, aquellos relacionados con los clientes (satisfacción, comunicación, imagen, etc.) y, finalmente, aquellos vinculados a medidas de rendimiento financiero y de mercado (ventas, beneficios y cuota de mercado).

La implicación de los clientes en el proceso de producción de un nuevo servicio se puede efectuar a través de distintas técnicas, unas más tradicionales que posiblemente con- 
duzcan a pequeñas modificaciones de los servicios actuales (Leonard y Rayport, 1997) y otras más innovadoras (observación, la etnografía ${ }^{3}$ o la utilización de lead users $^{4}$ ) que son capaces de detectar necesidades latentes (Carbonell et al., 2009). A través de esta implicación se puede obtener un producto final plenamente adaptado a las necesidades de los clientes y que presente una superioridad respecto a las ofertas competidoras. Además existen otros beneficios potenciales (Pelham y Wilson, 1996; Carbonell et al., 2009; Santos et al., 2009): (1) diseño de un producto de mayor calidad; (2) ajuste de tiempos; (3) percepción de la empresa como superior a la competencia y mejor imagen de la empresa en el mercado; (4) hallazgo de nuevos procesos para la provisión del servicio ("tensión creativa" entre empresa y clientes).

No obstante, el proceso no esta exento de limitaciones y posibles inconvenientes (Leonard, 1992; Magnusson et al., 2003; Carbonell et al., 2009; Santos et al., 2009): (1) los clientes aportan ideas que aunque pueden ser originales no siempre son factibles; (2) las ideas de los clientes no suelen conducir a grandes innovaciones; (3) la ausencia de mecanismos adecuados para transmitir dichas ideas y (4) los resultados no siempre tienen porqué ser favorables.

Considerando de forma conjunta los inconvenientes y las ventajas, los trabajos de Matthing et al. (2004), Alam (2006) y Carbonell et al. (2009) concluyen que la interacción empresa-consumidor es favorable, entre otros, por los siguientes motivos: proporciona una idea más completa de lo que los consumidores quieren, desean o necesitan, reduce el tiempo de desarrollo de un nuevo servicio y se produce una mejora en el rendimiento económico-financiero del nuevo servicio. A todas estas consideraciones podemos añadir, desde la LDS, que mediante la co-producción de innovaciones la empresa puede ampliar el ámbito de interacción con el cliente en el que puede actuar como co-creadora de valor. Por ello entendemos que la valoración de la participación de los clientes debería repercutir favorablemente en su lealtad, satisfacción, imagen de la empresa y percepción de valor añadido, variables que en este estudio representan los resultados de los clientes. A tal efecto se plantea la siguiente hipótesis:

H3: La predisposición cultural de las empresas hoteleras a la participación activa de sus clientes en la innovación de servicios ejerce un efecto positivo en los resultados de clientes respecto a la competencia.

Estudios empíricos previos han demostrado que la toma de decisiones participativa en las empresas mejora la motivación y satisfacción en el trabajo de sus empleados lo cual tiene su reflejo en la mejora de los resultados organizativos (Brewer et al., 2000; Bansal, et al., 2001; Kim, 2002; Ahmed et al., 2003).

En este sentido, el cliente se ve favorecido de forma indirecta, pero efectiva, por la aplicación del MI en la organización ya que ésta supone el desarrollo de una mentalidad del servicio en los empleados y de comportamiento orientado al cliente, que fomentan la entrega de un servicio superior creando, de este modo, clientes satisfechos, y construyendo

\footnotetext{
${ }^{3}$ Utiliza la observación singularizada del consumidor para ahondar en su comportamiento; se pretenden detectar costumbres o tendencias que expliquen las necesidades y preferencias del consumidor.

${ }^{4}$ Se utiliza un grupo de consumidores generalmente pioneros en el consumo de innovaciones y que tratan de anticipar las necesidades que posteriormente serán generalizadas para el mercado global.
} 
relaciones con el cliente (Berry, 1999; Gwinner et al., 2005; Cadwallader et al., 2010). Asimismo, las entidades que practican una gestión participativa permiten a sus empleados de primera línea reducir las posibles fuentes de tensión y el conflicto con los clientes durante el encuentro, redundando en una mayor satisfacción para el personal de contacto y los propios clientes (Jong et al., 2004; Gounaris, 2008).

Además, la involucración de los empleados de primera línea en el desarrollo de nuevos servicios también puede tener repercusión directa en la satisfacción de los clientes: (1) los empleados son los encargados de prestar el servicio y, por tanto, el haber participado en su diseño les aporta una mayor cualificación para el éxito de ejecución (Cadwallader et al., 2010); (2) cuando el servicio es complejo, la relación entre proveedor del servicio y cliente es clave (Crosby et al., 1990) de modo que los empleados de primera línea que han co-creado el nuevo servicio están en mejor situación para explicar las innovaciones de servicio a sus clientes y favorecer su introducción en el mercado; (3) las innovaciones de servicio guiadas por los empleados de contacto con los clientes pueden recoger en mayor medida las exigencias de los clientes, tanto actuales como futuras (Santos et al., 2009) y, finalmente, (4) la participación activa de los empleados en la innovación de servicios no sólo favorece mayores tasas de innovación sino que además contribuye a proteger dicha innovación, mediante la adhesión y lealtad de los empleados (Comisión UE, 2009) lo que sitúa a la empresa en condiciones de ofrecer mayor valor añadido a largo plazo. Por tanto, la cuarta hipótesis de la investigación establece que:

H4: La predisposición cultural de las empresas hoteleras a la participación activa de sus empleados de primera línea en la innovación de servicios ejerce un efecto positivo en los resultados de clientes respecto a la competencia.

Por último, la mejora de la respuesta del cliente llevará a que el hotel pueda obtener mejores resultados financieros (incremento de las ventas, cuotas de mercado, beneficios, etc.). Así, la mayor satisfacción y lealtad de los clientes permiten incrementar las ventas, reducir los costes, incrementar su eficiencia y eficacia y obtener una comunicación boca-oreja positiva por parte de los clientes (Fournier, et al., 1998; Rowe y Barnes, 1998; Ahmed et al., 2003; Sanzo et al., 2007). En consecuencia, la quinta hipótesis de la investigación se plantea como sigue:

H5: Los resultados de clientes afectan directa y positivamente a los resultados empresariales de las empresas hoteleras.

La Figura 2 resume las hipótesis del trabajo. Se propone un modelo en el que los efectos del MI se trasladan a través de distintas variables mediadoras hasta llegar al rendimiento empresarial. El MI se refleja como un elemento esencial para favorecer la aceptación del papel co-creador de clientes y empleados, el cual redunda en los mejores resultados de clientes y, finalmente, en la mejora de los resultados empresariales.

\section{METODOLOGÍA}

\section{1. Ámbito de estudio y características de la muestra analizada}

Para la realización del estudio empírico se recurrió a la base de datos SABI (actualización junio 2009). La delimitación de la población objetivo se llevó a cabo a partir de un cri- 
terio geográfico, empresas localizadas en la mitad norte de España, seleccionando hoteles con categoría de tres, cuatro y cinco estrellas, con al menos 10 trabajadores $^{5}$.

Figura 2

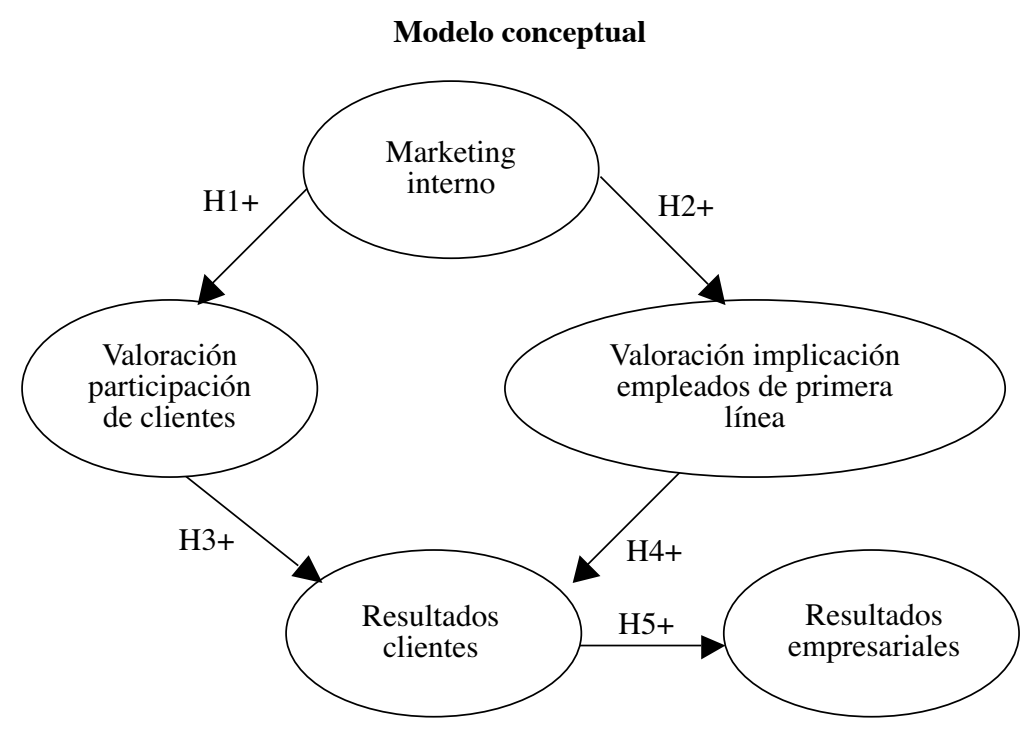

La industria hotelera resulta un escenario apropiado ya que, en los hoteles, muchas de las actividades de servicio son de primera línea y favorecen las co-producciones entre empleados y clientes. Como afirman Veflen y Sallis (2006:473) "mientras algunas partes del servicio son separables, como la limpieza de las habitaciones, en general la estancia del hotel es inseparable del servicio. Además, existe una alta variedad en el proceso de desarrollo del servicio entre los diferentes hoteles". Estas razones justifican el ámbito de estudio elegido para desarrollar el trabajo presentado.

Teniendo en cuenta los criterios anteriores, ámbito geográfico y categoría del hotel, se identificó una población de 1.481 empresas hoteleras. Tras contactar telefónicamente con los hoteles seleccionados, para comprobar la exactitud de los datos disponibles y solicitar la participación en el estudio del informante clave, la población finalmente analizada ascendió a 1.238 hoteles. La recogida de información se llevó a cabo mediante una encuesta dirigida a los gerentes de estas empresas. Se consideró oportuno que el informante fuese el máximo responsable de la gestión para garantizar la validez del contenido del estudio, puesto que se le presupone un conocimiento adecuado de todas las variables analizadas (Thorpe y Morgan, 2007).

\footnotetext{
${ }^{5}$ Se controló contar con información de sólo un hotel por cadena hotelera, dado que entendemos que las empresas de un mismo grupo comparten su estrategia de gestión.
} 
La muestra finalmente obtenida consta de 240 hoteles (tasa de respuesta del 19,4\%) de las cuales, un 57,5\% pertenece a la categoría de tres estrellas, un 37,9\% a la de cuatro estrellas y el 4,6\% restante a la de cinco estrellas. El número medio de empleados por hotel es de 33 personas.

\subsection{Medición de las variables del modelo}

Se solicitó a los entrevistados que indicasen, en una escala tipo Likert de siete posiciones, su grado de acuerdo o desacuerdo con las afirmaciones planteadas ( $1=$ total desacuerdo; 7=total acuerdo) para medir los conceptos culturales analizados en este estudio. La redacción de las escalas se recoge en el Anexo y éstas fueron previamente discutidas y valoradas en un pre-test desarrollado con cuatro hoteles. En cuanto a las escalas de resultados tanto de clientes como empresariales, siguiendo los trabajos de Avlonitis y Gounaris (1999) y Theoharakis y Hooley (2003), se consideró conveniente pedirles a los encuestados que valorasen en qué grado les parecía que su empresa había conseguido alcanzar esos resultados en los últimos tres años. Se ha recurrido al mismo tipo de escala (Likert), en la que 1 significa "de ningún modo" (se ha alcanzado dicho resultado) y 7 "de modo muy importante". En la Tabla 1 se muestran las principales características de las escalas utilizadas.

Tabla 1

\section{Características de las escalas empleadas para la obtención de la información}

\begin{tabular}{lcc}
\hline & $\begin{array}{c}\text { Número } \\
\text { de Items }\end{array}$ & Fuentes \\
\hline $\begin{array}{l}\text { Marketing interno } \\
\begin{array}{l}\text { Predisposición a la partici- } \\
\text { pación activa de los clientes }\end{array}\end{array}$ & 16 & Lings (2004); Gounaris (2005); Sanzo et al., (2007) \\
$\begin{array}{l}\text { Participación de los } \\
\text { empleados de primera línea }\end{array}$ & 5 & $\begin{array}{c}\text { De Brentani y Ragot (1996); Hays y Hill (2001); Bate- } \\
\text { son (2002); Sharpley y Forster (2003) }\end{array}$ \\
$\begin{array}{l}\text { Resultados de clientes } \\
\text { Resultados empresariales }\end{array}$ & 7 & $\begin{array}{c}\text { Rafiq y Ahmed (2000); Lings (2004); Zahay y Griffin } \\
\text { (2004); Gounaris (2005); Hooley et al. (2005); Vorhies } \\
\text { y Morgan (2005) }\end{array}$ \\
$\begin{array}{l}\text { Theoharakis y Hooley (2003); Tippins y Sohi (2003); } \\
\text { Vorhies y Morgan (2005); Weerawardena } \text { et al. (2006) }\end{array}$ \\
\hline
\end{tabular}

\subsection{Resultados}

El análisis de los resultados se realiza en dos fases. En primer lugar, se evalúan las propiedades psicométricas (fiabilidad, validez convergente y discriminante) de cada una de las escalas de medida empleadas de acuerdo con las sugerencias metodológicas de Churchill (1979) y Anderson y Gerbing (1988). Seguidamente, se procede a contrastar las hipótesis 
recogidas en el modelo conceptual mediante un sistema de ecuaciones estructurales. Para ello se recurre al paquete estadístico EQS 6.1 para Windows en el que se emplea el método de estimación de máxima verosimilitud robusto.

\section{Fiabilidad y validez de las escalas de medida}

El análisis de la fiabilidad de las escalas se comprueba examinando que, en todos los casos, el coeficiente de fiabilidad compuesto sea superior al valor recomendado de 0,6 y que la varianza media extraída (AVE) supere el mínimo establecido de 0,5 (Hair et al., 1999). La validez convergente se evalúa comprobando que los parámetros lambda estandarizados sean siempre significativos y superiores al valor 0,5 (Hildebrandt, 1987; Gerbing y Anderson, 1988; Steenkamp y Van Trijp, 1991). Para determinar la validez discriminante entre las escalas propuestas, se comprobó que la raíz cuadrada del AVE de cada constructo supera la covarianza entre éste y cualquier otro del modelo, es decir, que la varianza media compartida entre un concepto y sus medidas es superior a la varianza compartida entre constructos (Fornell y Larker, 1981).

El paso previo consistió en comprobar la fiabilidad y validez (convergente y discriminante) de la escala de MI, así como la convergencia adecuada de las tres dimensiones en un único factor. En el Anexo que se recoge al final del trabajo figuran los ítems que fueron eliminados. Las Tablas 2 y 3 muestran los resultados obtenidos en tales análisis así como los índices de bondad del ajuste.

Tabla 2

Fiabilidad de las dimensiones de marketing interno

\begin{tabular}{lcccc}
\hline \multicolumn{1}{c}{ Dimensiones } & Item & $\begin{array}{c}\text { Cargas factoriales } \\
\text { (valor t) }\end{array}$ & $\begin{array}{c}\text { Coeficiente de } \\
\text { Fiabilidad }\end{array}$ & AVE \\
\hline GENERACIÓN (GEN) & GEN1 & $0,65(7,90)$ & 0,834 & 0,630 \\
& GEN5 & $0,84(13,00)$ & & \\
& GEN6 & $0,87(13,60)$ & 0,834 \\
DISEMINACIÓN (DIS) & DIS2 & $0,91(10,34)$ & 0,938 & \\
& DIS3 & $0,93(10,94)$ & & \\
& DIS4 & $0,90(9,62)$ & \\
RESPUESTA (RESP) & RESP4 & $0,78(8,65)$ & 0,827 \\
& RESP5 & $0,88(11,83)$ & \\
& RESP6 & $0,68(10,79)$ & \\
\hline S-B $\chi 2(24)=45,9087$ & Medidas de Bondad del Ajuste & & \\
$(\mathrm{p}=0,00454)$ & BBNNFI & CFI & \\
\end{tabular}


Tabla 3

Validez discriminante de las dimensiones de marketing interno

\begin{tabular}{lccc}
\hline & GEN & DIS & RESP \\
\hline GEN & 0,794 & --- & --- \\
DIS & 0,653 & 0,913 & --- \\
RESP & 0,739 & 0,759 & 0,785 \\
\hline
\end{tabular}

Nota: Los elementos de la diagonal representan la raíz cuadrada del AVE de cada una de las escalas. Los elementos situados fuera de la diagonal corresponden a las correlaciones entre cada par de escalas.

En las Tablas 4 y 5 se recogen los resultados del procedimiento descrito para el modelo global. Tal y como se puede observar en la Tabla 4, el modelo factorial confirmatorio ofrece unas medidas de calidad del ajuste adecuadas. También se constata que los valores alcanzados por los coeficientes de fiabilidad compuestos y los parámetros lambda estandarizados confirman la fiabilidad y validez convergente de las escalas incluidas en el modelo. Por último, los resultados recogidos en la Tabla 5 demuestran además la existencia de validez discriminante entre las escalas propuestas.

Tabla 4

Fiabilidad de las escalas

\begin{tabular}{lcccc}
\hline \multicolumn{1}{c}{ Dimensiones } & Item & $\begin{array}{c}\text { Cargas factoriales } \\
\text { (valor t) }\end{array}$ & $\begin{array}{c}\text { Coeficiente de } \\
\text { Fiabilidad }\end{array}$ & AVE \\
\hline MARKETING INTERNO & MI1 & $0,78(10,33)$ & 0,847 & 0,650 \\
(MI) & MI2 & $0,78(7,58)$ & & \\
& MI3 & $0,85(11,34)$ & & \\
IMPLICACIÓN EMPLEADOS & PRILINEA1 & $0,79(7,62)$ & 0,910 & 0,669 \\
PRIMERA LÍNEA (PRILINEA) & PRILINEA2 & $0,85(9,11)$ & & \\
& PRILINEA3 & $0,85(7,93)$ & & \\
& PRILINEA4 & $0,75(9,66)$ & & \\
& PRILINEA5 & $0,84(10,35)$ & & \\
& & & & \\
PARTICIPACIÓN ACTIVA & MOTIV1 & $0,61(8,27)$ & 0,849 & \\
CLIENTES (MOTIV) & MOTIV2 & $0,85(16,78)$ & & 0,746 \\
& MOTIV3 & $0,92(17,75)$ & & \\
& MOTIV5 & $0,65(9,75)$ & & \\
RESULTADOS CLIENTES & RCL1 & $0,91(12,56)$ & 0,921 & \\
(RCL) & RCL2 & $0,83(10,20)$ & & \\
& RCL3 & $0,89(14,87)$ & & \\
& RCL4 & $0,83(10,86)$ & & \\
RESULTADOS & RE1 & $0,96(19,04)$ & 0,943 \\
EMPRESARIALES (RE) & RE2 & $0,88(14,18)$ & & \\
& RE3 & $0,91(17,25)$ & & \\
\hline
\end{tabular}




\begin{tabular}{lccc}
\hline \multicolumn{4}{c}{ Medidas de Bondad del Ajuste } \\
\hline $\mathrm{S}-\mathrm{B} \chi 2(142)=219,8584$ & BBNNFI & CFI & RMSEA \\
$(\mathrm{p}=0,00003)$ & 0,943 & 0,953 & 0,048 \\
\hline
\end{tabular}

Tabla 5

Validez discriminante de las escalas

\begin{tabular}{lccccc}
\hline & MKI & PRILINEA & MOTIV & RCL & RE \\
\hline MKI & 0,806 & --- & --- & --- & --- \\
PRILINEA & 0,445 & 0,818 & --- & --- & --- \\
MOTIV & 0,270 & 0,263 & 0,769 & --- & --- \\
RCL & 0,563 & 0,448 & 0,225 & 0,864 & --- \\
RE & 0,347 & 0,352 & 0,264 & 0,498 & 0,918 \\
\hline
\end{tabular}

Nota: Los elementos de la diagonal representan la raíz cuadrada del AVE de cada una de las escalas. Los elementos situados fuera de la diagonal corresponden a las correlaciones entre cada par de escalas.

\section{Estimación del modelo causal}

Para la estimación del modelo causal se utilizó nuevamente la técnica de las ecuaciones estructurales y el paquete estadístico EQS para Windows 6.1 (Tabla 6). Como se puede observar los resultados de este estudio confirman que el MI ejerce un efecto directo y significativo tanto en la disposición de las empresas para la mayor participación de los empleados de primera línea en el desarrollo de nuevos servicios (H2), como en el deseo de participación activa de los clientes en las innovaciones (H1). La mayor predisposición a la participación de ambos colectivos (empleados y clientes) en el desarrollo de innovaciones ejerce un efecto positivo y significativo sobre los resultados de clientes (H4 y H3) que obtienen los hoteles analizados respecto a sus competidores, lo que en última instancia se traduce en el logro de ventajas competitivas en términos de resultados empresariales (H5).

Tabla 6

Efectos del marketing interno, la implicación de los empleados y de los clientes en los resultados de las empresas hoteleras

\begin{tabular}{lcc}
\hline \multicolumn{1}{c}{ Hipótesis } & $\begin{array}{c}\text { Parámetro } \\
\text { (valor } \mathrm{t})\end{array}$ & Resultado \\
\hline H1: MI $\rightarrow$ Valoración participación activa clientes & $0,29(3,58)$ & Sig. \\
\hline H2: MI $\rightarrow$ Valoración implicación empleados primera línea & $0,47(4,03)$ & Sig. \\
\hline H3: Valoración participación activa clientes $\rightarrow$ Resultados clientes & $0,13(1,69)$ & Sig.* \\
\hline H4: Valoración implicación empleados primera línea $\rightarrow$ Resultados clientes & $0,44(4,86)$ & Sig. \\
\hline H5: Resultados clientes $\rightarrow$ Resultados empresariales & $0,50(9,03)$ & Sig. \\
\hline
\end{tabular}

Sig. = Relación significativa al 0,05

*Sig. = Relación significativa al 0,1

Medidas de bondad del ajuste $=\chi^{2}(147)=256,2841 ; \mathrm{p}=0,000 ; \mathrm{BBNNFI}=0,923 ; \mathrm{CFI}=0,934 ; \mathrm{RMSEA}=0,056$ 


\section{CONCLUSIONES, LIMITACIONES Y FUTURAS LÍNEAS DE INVESTIGACIÓN}

En este trabajo se analiza el impacto del MI, o de la gestión de los recursos humanos como un mercado interno al que es necesario proporcionar valor (Gounaris, 2006), en los resultados competitivos de las empresa hoteleras. Para alcanzar este objetivo se plantea el papel mediador en la relación MI-resultados de dos variables: la predisposición cultural de las empresas hoteleras a colaborar con sus clientes en el desarrollo de nuevos servicios turísticos, y dicha predisposición a la colaboración con los empleados de primera línea. Estas dos variables se han identificado en la literatura reciente como relevantes para el desarrollo de innovaciones conjuntas con éxito en el sector servicios dado, que como paso previo a la colaboración efectiva en la co-creación de nuevos servicios, se necesita valorar la existencia de dicha colaboración (Carbonell et al., 2009). Los resultados de esta investigación confirman que la aplicación del MI en los hoteles ejerce un efecto positivo en ambas variables mediadoras, aunque más importante en la valoración de la co-creación de innovaciones con los empleados de primera línea en $(0,47)$ que en la valoración de la co-creación con clientes $(0,29)$. En este sentido, el MI suscita otras subculturas favorables no sólo a la innovación sino también a que ésta sea compartida con clientes y empleados; de este modo, tal y como se planteaba al principio de este estudio, podemos comprender con mayor detalle los efectos del MI en la gestión empresarial. El MI, como estrategia de gestión de los recursos humanos, ejerce no obstante un efecto más fuerte sobre la valoración de los empleados.

Siguiendo en la misma línea argumental, la predisposición favorable de las empresas hoteleras a considerar la involucración de sus clientes en el proceso de innovación conduce a la consecución de mejores resultados con éstos $(0,13)$. Este efecto positivo se sustenta en la posibilidad de desarrollar innovaciones de servicio más adaptadas a las necesidades de los clientes y a reducir las posibilidades de fracaso. La valoración de la participación de los empleados de primera línea en los procesos de innovación permite no sólo incorporar sus sugerencias, sino adaptar también la oferta a la evolución de las necesidades del mercado y situarlos en mejor posición para ejecutar y favorecer la difusión de las innovaciones de servicio; por todo ello también se confirman los efectos positivos de esta subcultura en los resultados de clientes de las empresas hoteleras. Es preciso resaltar, no obstante, que el efecto de la valoración de la co-creación con empleados en términos de grado de satisfacción, lealtad y comunicación con los clientes y valor añadido percibido, es mayor $(0,44)$ que en el caso de la valoración de co-creación con los propios clientes. Estos datos, refuerzan el papel esencial de los empleados en las empresas hoteleras y la importancia de su gestión eficiente para lograr una mayor competitividad a largo plazo máxime, cuando los resultados del estudio también confirman que los resultados de clientes permiten a los hoteles, en última instancia, mejorar su posición competitiva en el mercado en términos de ventas, beneficios y cuota de mercado $(0,50)$.

Dado que todos los indicadores de resultados se midieron con una referencia temporal de tres años, podemos concluir que aquellas empresas hoteleras que aplican MI y que, como consecuencia, valoran en mayor medida la participación de sus clientes y empleados de primera línea en la co-creación de nuevos servicios, obtienen de forma consistente mejores resultados que sus competidores con sus clientes, en indicadores de mercado y financieros, lo que sugiere el logro de ventajas competitivas de forma sostenible. 
La escala de MI, aplicada con éxito en estudios anteriores entre empresas manufactureras, permite tan sólo la supervivencia de 9 ítems en el sector turístico. Observando los bloques de variables que han sido eliminados se puede comprobar que, en relación a la generación de información sobre el mercado interno, la escala no mide el grado en que los hoteles de la muestra conocen las políticas de personal de su competencia, los competidores que pueden atraer a sus empleados clave y las condiciones exactas del mercado laboral. Así mismo, tampoco se recoge en la respuesta a la información interna si el hotel dispone de una política de diseño de puestos de acuerdo con las capacidades y desarrollo profesional de sus empleados y de un plan de formación estable.

La justificación de estos resultados podemos encontrarla en el propio informe sobre el sector turístico en Europa publicado en septiembre de 2009 por la Comisión UE del que se puede inferir que muchas empresas hoteleras carecen de una adecuada orientación externa que puedan aplicar a la gestión de su personal y pueden adolecer de falta de conocimientos de gestión adecuados para desarrollar una política de recursos humanos basada en el diseño de puestos, la carrera profesional y la formación regular.

Entre las líneas de investigación futuras que se derivan de este trabajo podemos señalar tres aspectos esenciales. Por un lado, es preciso profundizar en las políticas de recursos humanos que se desarrollan en las empresas hoteleras reformulando la medición del concepto de MI en estas organizaciones. En segundo lugar, puede ser oportuno introducir en el modelo otras variables que puedan actuar como antecedentes de las culturas de co-creación de innovaciones, tales como la propia orientación al mercado o la cultura innovadora, comparando su efecto con el del MI. Finalmente, el análisis desarrollado a nivel organizativo es necesario trasladarlo al estudio de los efectos del MI en la co-creacion de nuevos servicios concretos con clientes y empleados de primera línea.

Entre las limitaciones del trabajo se pueden destacar las siguientes. Primero, la información sobre las variables independientes y dependiente se ha recogido en un mismo momento del tiempo y de un mismo informante, con lo que es posible que se de un problema de sesgo debido al procedimiento empleado o common method bias. No obstante, cabe señalar, por un lado, que este tipo de problema es más probable cuando se miden constructos que implican fuertes sentimientos, como las actitudes, mientras que en el caso de las variables de resultado como las utilizadas es menos probable que aparezca (Chen $e t$ al., 2005). Además, en este trabajo se ha tomado la precaución de incluir una "separación psicológica" dentro del cuestionario entre la parte en la que se interrogaba al encuestado por el MI y la cultura de co-creación con clientes y empleados y aquella en la que se le solicitaban los datos de resultados (Podsakoff et al., 2003). Esta separación supuso incluir otras cuestiones que facilitaban el hecho de que el encuestado no vinculara directamente las variables predictora y dependiente. Una limitación adicional se deriva de la utilización de medidas subjetivas de resultados financieros, si bien se trata de una práctica corriente en las investigaciones ante la resistencia que muestran las empresas a proporcionar datos cuantitativos de ventas, cuotas de mercado o beneficios. 


\section{REFERENCIAS BIBLIOGRÁFICAS}

AHMED, P.K.; RAFIQ, M. Y SAAD, N.M. (2003): "Internal marketing and the mediating role of organizational competencies", European Journal of Marketing, Vol. 37, No. 9, pp. 1221-1241.

ALAM, I. (2002): "An exploratory investigation of user involvement in new service development", Journal of Academy of Marketing Science, Vol. 30, No. 3, pp. 250-261.

ALAM, I. (2005): "Removing the fuzziness from the fuzzy front-end of service innovations through customer interactions", Industrial Marketing Management, Vol. 35, No. 4, pp. 468-480.

ALAM, I. (2006): "Service innovation strategy and process: A cross-national comparative analysis", International Marketing Review, Vol. 23, No. 3, pp. 234-254.

ANDERSON, J.C. Y GERBING, D.W. (1988): "Structural equation modelling in practice: A review and recommended two-step approach", Psychological Bulletin, Vol. 103, No. 3, pp. 411-423.

ARNDT, J. (1983): “The political economy paradigm: Foundation for theory in Marketing", Journal of Marketing, Vol. 47, pp. 44-54.

AVLONITIS, G.J. Y GOUNARIS, S.P. (1999): "Marketing orientation and its determinants: An empirical analysis", European Journal of Marketing, Vol. 33, No. 11/12, pp. 1003-1037.

BALLANTYNE D.; VAREY, R.J.; FROW, P. Y PAYNE, A. (2008): "Service-dominant logic and value propositions: Re-examining our mental models", Otago Forum 2 - Academic Papers, Otago, New Zealand.

BANSAL, H.S.; MENDELSON, M.B. Y SHARMA, B. (2001): "The impact of internal marketing activities on external marketing outcomes", Journal of Quality Management, Vol. 6, pp. 61-76.

BARNES, J.G. (1989): “The internal marketing programme: If the staff won't buy it, why should the customer?", Proceedings of the Marketing Education Group Annual Conference, Glasgow, pp. 453-474.

BATESON, J. (2002): “Consumer performance and quality in services”, Managing Service Quality, Vol. 12, No. 4, pp. 206-209.

BERRY, L.L. (1981): “The employee as customer”, Journal of Retail Banking, Vol. 3, No. 1, pp. 33-40.

BERRY, L.L. (1999): Discovering the soul of service, New York: Free Press.

BREWER, G.A.; SELDEN, S.C. Y FACER, R.L. (2000): "Individual conceptions of public services motivation", Public Administration Review, Vol. 60, No. 3, pp. 254-263.

CADWALLADER, S; BURKE, C.; BITNER, M. Y O.A. (2010): "Frontline employee motivation to participate in service innovation implementation", Journal of the Academy Marketing Science, Vol. 38, pp. 219-239.

CARBONELL FOULQUIÉ, P.; RODRÍGUEZ ESCUDERO, A.I. Y PUJARI, D. (2009): "Customer involvement in new service development: An examination of antecedents and outcomes", Journal of Product Innovation Management, Vol. 29, No. 5, pp. 536-550.

CHEN, J.; REILLY, R. R. Y LYNN, G. S. (2005): “The impact of speed-to-market on new product success: The moderating effects of uncertainty", IEEE Transactionson Engineering Management, Vol. 52, No2, pp. 199-212.

COMISIÓN UE (2009): Estudio sobre la competitividad en el sector turístico de la UE, Directorate-General Enterprise \& Industry.

CONDUIT, J. Y MAVONDO, F.T. (2000): "Market orientation: Implications for employee perceptions", Congreso ANZMAC 2000 Visionary Marketing for the $21^{\text {st }}$ Century: Facing the Challenge, pp. 197-201.

CROSBY, L.A.; EVANS, K.R. Y COWLES, D. (1990): "Relationship quality in services selling: An interpersonal influence perspective", Journal of Marketing, Vol. 54, No 3, pp. 68-81.

CHURCHILL, G. (1979): "A paradigm for developing better measures of marketing constructs", Journal of Marketing Research, Vol. 16, pp. 64-73. 
DE BRENTANI, U. Y RAGOT, E. (1996): "Developing new business-to-business professional services: What factors impact on performance”, Industrial Marketing Management, Vol. 25, pp. 517530.

DOMÍNGUEZ, C.; MARTÍN, J.D. Y DE SAÁ, P. (2009): "El desarrollo de una cultura de orientación al cliente interno a través de las prácticas de recursos humanos de alto compromiso: Un modelo explicativo", XIX Congreso Nacional de ACEDE, Toledo.

FORNELL, C. Y LARKER, D.F. (1981): "Evaluating structural equation models with unobservable variables and measurement errors", Journal of Marketing Research, Vol. 18, pp. 39-50.

FOURNIER, S.; DOBSCHA, S. Y MICK, D.G. (1998): "Preventing the premature death of relationship marketing," Harvard Business Review, Vol. January-February, pp. 6-21.

GEORGE, W.R. (1990): Internal marketing organizational behaviour: A partnership in developing customer-conscious employees at every level", Journal of Business Research, Vol. 20, pp. 63-70.

GERBING, D.W. Y ANDERSON, J.C. (1988): "An updated paradigm for scale development incorporating unidimensionality and its assessment", Journal of Marketing, Vol. 55, No. October, pp. $1-19$.

GOUNARIS, S.P. (2005): "Trust and commitment influences on customer retention: Insights from business-to-business services", Journal of Business Research, Vol. 58, No.2, pp.126-40.

GOUNARIS, S.P. (2006): "Internal-market orientation and its measurement", Journal of Business Research, Vol. 59, No. 4, pp. 432-448.

GOUNARIS, S.P. (2008): "Antecedents of internal marketing practice: Some preliminary empirical evidence", International Journal of Service Industry Management, Vol. 19, No. 3, pp. 400-434.

GRÖNROOS, C. (1981): "Internal marketing-an integral part of marketing theory", in Donnelly, J.H. and George, W.E. (Eds.), Marketing of Services, American Marketing Association Proceedings Series, pp. 236-238.

GRÖNROOS, C. (2008): “Service Logic Revisited: Who Creates Value? And Who Co-Creates?", European Management Review, Vol. 20, No. 4, pp. 298-314.

GWINNER, K.; BITNER, M.J.; BROWN, S.W. Y KUMAR, A. (2005): "Service customization through employee adaptiveness", Journal of Service Research, Vol. 8, No. 2, pp.131-148.

HAIR, J.; ANDERSON, R.; TATHAM, R. Y BLACK, W. (1999): Análisis Multivariante, quinta edición, ed. Prentice Hall, Madrid.

HAYS, J. Y HILL, A. (2001): "A preliminary investigation of the relationships between employee motivation/vision, service learning, and perceived service quality", Journal of Operations Management, Vol. 19, No. 3, pp. 335-349.

HILDEBRANDT, L. (1987): "Consumer retail satisfaction in rural areas: A reanalysis of survey data", Journal of Economic Psychology, Vol. 8, pp. 19-42.

HOOLEY, G.J.; GREENLEY, G.E.; CADOGAN, J.W. Y FAHY, J. (2005): “The performance impact of marketing resources", Journal of Business Research, Vol. 58, pp. 18-27.

INE (2009). Instituto Nacional de Estadística, www.ine.es.

JONG, A.; DE RUYTER, K. Y DE LEMNIK, J. (2004): "Antecedents and consequences of services climate in boundary-spanning self-managing service teams", Journal of Marketing, Vol. 68, No. 1, pp. 18-35.

KELEMEN, M. Y PAPASOLOMOU-DOUKAKIS, I. (2004): "Can culture be changed? A study of internal marketing", The Service Industries Journal, Vol. 24, No 5, pp. 121-135.

KIM, S. (2002): "Participative management and job satisfaction: Lessons for management leadership", Public Administration Review, Vol. 62, pp. 231-241.

KOHLI, A.K. Y JAWORSKI, B.J. (1990): "Market orientation: The construct, research propositions, and managerial implications", Journal of Marketing, Vol. 54, No. Abril, pp. 1-18.

LEONARD BARTON, D. Y RAYPORT, J. (1997): "Spark innovation through empathic design", Harvard Business Review, Vol. 75, No. 6, pp. 102-13. 
LINGS, I.N. (2004): "Internal market orientation: Constructs and consequences", Journal of Business Research, Vol. 57, No. 4, pp. 405-413.

LINGS, I.N. Y GREENLEY, G.E. (2005). Measuring internal market orientation, Journal of Service Research, Vol. 7, No. 3, 290-306.

LUSCH, R.F.; VARGO, S.L. Y TANNIRU, M. (2009): "Service, value networks and learning", Journal of the Academy of Marketing Science, Vol. 38, No. 1, pp. 19-31.

LEONARD, D. (1992): "Core capabilities and core rigidities: A paradox in managing new product development", Strategic Management Journal, Vol. 13, No February, pp. 111-125.

MAGNUSSON, P.R.; MATTHING, J. Y KRISTENSSON, P. (2003): "Managing user involvement in service innovation", Journal of Service Research, Vol. 6, No. 2, pp. 111-124.

MATTHING, J.; SANDÉN, B. Y EDVARDSSON, B. (2004): "New service development: Learning from and with customers", International Journal of Service Industry Management, Vol. 15, No. 5, pp. 479-498.

NASUTION, H.N. Y MAVONDO, F. (2008): "Customer value in the hotel industry: What managers believe they deliver and what customer experience", International Journal of Hospitality Management, Vol. 27, pp. 204-213.

NAUDÉ, P.; DESAI, J. Y MURPHY, J. (2003): "Identifying the determinants of internal marketing orientation", European Journal of Marketing, Vol. 37, No. 9, pp. 1205-1220.

O’REILLY, C. A. III, Y PFEFFER, J. (2000): Hidden value: How great companies achieve extraordinary results with ordinary people, Boston, MA: Harvard Business School Press.

PELHAM, A. M. Y WILSON, D. T. (1996): "A longitudinal study of the impact of market structure, firm structure, strategy, and market orientation culture on dimensions of small-firm performance", Journal of the Academy of Marketing Science, Vol. 24, No. 1, pp. 27-43.

PODSAKOFF, P.; SCOTT, B.; LEE, J. Y PODSAKOFF, N. (2003): "Common method biases in behavioural research: A critical review of the literature and recommended remedies", Journal of Applied Psychology, Vol. 88, pp. 879-903.

RAFIQ, M. Y AHMED, P.K. (1993): "The scope of internal marketing: defining the boundary between marketing and human resource management", Journal of Marketing Management, Vol. 9, pp. 219-232.

RAFIQ, M. Y AHMED, P.K. (2000): "Advances in the internal marketing concept: Definition, synthesis and extension", Journal of Services Marketing, Vol. 14, No 6, pp. 449-462.

ROWE, W.G. Y BARNES, J.G. (1998): "Relationship marketing and sustained competitive advantage," Journal of Market-Focused Management, Vol. 2, No. 3, pp. 281-297.

SAN MARTÍN, S. (2005): "El marketing interno como herramienta clave en la gestión de RR.HH.", Dirigir Personas, Vol. 34, No. Junio, pp. 32-45.

SANTOS, M.L. Y ÁLVAREZ, L.I. (2007). "Innovativeness and organizational innovation in total quality oriented firms: The moderating role of market turbulence", Technovation Vol. 27, No. 9, pp. 514-532.

SANTOS, M.L.; GONZÁLEZ, C. Y LÓPEZ, J.A. (2009): “Cultura innovadora, co-creación y resultados en las KIBS", XXI Congreso Nacional de Marketing, Bilbao.

SANZO, M.J.; GARCÍA, N.; SANTOS, M. L. Y TRESPALACIOS, J. A. (2007): "El marketing interno como fuente de ventaja competitiva para las PYME: Repercusiones sobre diversos tipos de resultados", XXI Congreso Nacional y XVII Congreso Hispano-Francés de la Academia Europea de Dirección y Economía de Empresa, Universidad Rey Juan Carlos, Madrid.

SASSER, W.E. Y ARBEIT, S.F. (1976): "Selling jobs in the service sector", Business Horizons, Vol. 19, June, pp. 61-65.

SHARPLEY R. Y FORSTER G. (2003): "The implications of hotel employee attitudes for the development of quality tourism: The case of Cyprus", Tourism Management, Vol. 24, No. 6, pp. 687-697. 
SHIU, Y.M. Y YU, T.W. (2010): "Internal marketing, organisational culture, job satisfaction, and organisational performance in non-life insurance", The Service Industries Journal, Vol. 30, No. 6, pp. 793-809.

STEENKAMP, J.B. Y VAN TRIP, H. (1991): "The use of LISREL in validating marketing constructs", International Journal of Research in Marketing, Vol. 8, pp. 283-299.

THEOHARAKIS, V. Y HOOLEY, G. (2003): "Organizational resources enabling service responsiveness: Evidence from Greece”, Industrial Marketing Management, Vol. 32, pp. 695-702.

THORPE, E.R. Y MORGAN, R.E. (2007): "In pursuit of the ideal approach to successful marketing strategy implementation", European Journal of Marketing, Vol. 41, No. 5/6, pp. 659-677.

TIPPINS, M.J. Y SOHI, R.S. (2003): "It competency and firm performance: Is organizational learning a missing link?", Strategic Management Journal, Vol. 24, pp. 745-761.

TORTOSA-EDO, V., SÁNCHEZ-GARCÍA, J. Y MOLINER-TENA, M.A. (2010): "Internal market orientation and its influence on the satisfaction of contact personnel", The Service Industries Journal, Vol. 30, No. 8, pp. 1279-1297.

VASCONCELOS, A.F. (2008) "Broadening even more the internal marketing concept", European Journal of Marketing, Vol. 42, No. 11/12, pp.1246-1264.

VARGO, S.L. Y LUSCH, R.F. (2004): "Evolving to a new dominant logic for marketing", Journal of Marketing, Vol. 68, No. January, pp. 1-17.

VEFLEN, N. Y SALLIS, J. (2006): "Market scanning for new service development", European Journal of Marketing, Vol. 40, No. 5/6, pp. 466-484

VORHIES, D.W. Y MORGAN, N.A. (2005): "Benchmarking marketing capabilities for sustainable competitive advantage", Journal of Marketing, Vol. 69, No. January, pp. 80-94.

WAGNER, J.A. III (1994): "Participation's effect on performance and satisfaction: A reconsideration of research evidence", Academy of Management Review, Vol. 19, No. 2, pp. 312-330.

WEERAWARDENA, J.; CASS, A. Y JULIAN, C. (2006): "Does industry matter? Examining the role of industry structure and organizational learning in innovation and brand performance", Journal of Business Research, Vol. 59, pp. 37-45.

WINTER, J.P. (1985): "Getting your house in order with internal marketing: A marketing prerequisite", Health Marketing Quarterly, Vol. 3, No. 1, pp. 69-77.

WORLD TOURISM ORGANIZATION (2009): UNWTO Tourism Highlights, 2009 Edition, disponible en: www.world-tourism.org (10/07/2010)

ZAHAY, D. Y GRIFFIN, A. (2004): "Customer learning processes, strategy selection and performance in business-to-business service firms", Decision Sciences, Vol. 35, No. 2, pp.169-203. 


\section{ANEXO}

\section{MARKETING INTERNO}

\section{MKI1: Generación de información sobre el mercado interno}

GEN1= La dirección del hotel conoce las necesidades y expectativas laborales de sus empleados

GEN2 = Conocemos las políticas de personal que aplica la competencia

GEN3 = La dirección del hotel conoce las características del mercado laboral en su sector de actividad

GEN4= Sabemos cuáles son los hoteles que pueden atraer a nuestros empleados clave GEN5= Conocemos las necesidades laborales particulares de cada colectivo de empleados de nuestro hotel

GEN6= La política de personal de nuestro hotel tiene en cuenta las características de cada colectivo de empleados

\section{MKI2: Diseminación de la información interna}

$D I S 1=$ Los empleados informan sobre sus problemas personales cuando estos afectan a su rendimiento

DIS2= Los cargos directivos del hotel están dispuestos a escuchar los problemas de los trabajadores

DIS3 = Los cargos directivos se comunican y comparten los problemas que puedan existir con los empleados

DIS4= La dirección se informa de los problemas y/o dificultades que tienen los empleados en el desempeño de sus funciones

\section{MKI3: Respuesta a la información interna}

RESPI = El diseño de puestos se hace teniendo en cuenta las capacidades profesionales de los empleados

RESP2 $=$ El diseño de puestos tiene en cuenta el desarrollo profesional de los empleados RESP3 = El hotel dispone de un Plan de Formación para sus empleados

RESP4= El hotel actúa sobre la base de que sus empleados son su principal recurso

RESP5= La política de personal busca activamente mejorar la satisfacción y condiciones laborales de los empleados

RESP6 = Los empleados reciben formación relacionada con sus necesidades

VALORACIÓN DE LA PARTICIPACIÓN ACTIVA CLIENTES

MOTIV1= Los clientes son innovadores y permiten crear mejores servicios

MOTIV2= La participación de los clientes permite ahorrar dinero

MOTIV3= Permite reducir el tiempo de desarrollo de nuevos servicios

MOTIV4 = Es una forma de dar a conocer la empresa en el mercado

MOTIV5= Los servicios basados en ideas de los clientes se adaptan mejor a las necesidades del mercado

VALORACIÓN DE LA IMPLICACIÓN DE LOS EMPLEADOS DE PRIMERA LÍNEA PRILINEA1= Los empleados en contacto con los clientes (primera línea) son una fuente muy valiosa de ideas de nuevos servicios

PRILINEA2= La participación de los empleados de primera línea es esencial para captar las exigencias del mercados 
PRILINEA3= Los empleados de primera línea deben jugar un papel activo en el proceso de desarrollo de nuevos servicios

PRILINEA4= El criterio de estos trabajadores es muy importante para determinar el éxito de un nuevo servicio

PRILINEA5= Los empleados de primera línea disponen de conocimiento muy valioso para el desarrollo de nuevos servicios

\section{RESULTADOS CLIENTES}

RCL1 $=$ Mayor grado de satisfacción de nuestros clientes

RCL2= Mayor grado de lealtad de nuestros clientes, clientes que repiten y son regularmente fieles

RCL3 = Mayor valor añadido proporcionado a nuestros clientes

RCL4= Mayor grado de comunicación con nuestros clientes

RCL5 = Reducción del número de quejas y reclamaciones de nuestros clientes

RCL6= Mejora de la imagen de la empresa ante nuestros clientes

$R C L 7=$ Mayor retención de los mejores clientes del mercado

\section{RESULTADOS EMPRESARIALES}

$\mathrm{RE} 1=$ Crecimiento de las ventas

RE2 $=$ Crecimiento de la cuota de mercado

$\mathrm{RE} 3=$ Crecimiento de los beneficios

Nota: Los ítems en cursiva fueron eliminados 


\section{Gipuzkoaren kanpo harremanak, esku onetan.}

Las relaciones externas de Gipuzkoa, en buenas manos.

Les relations externes de Gipuzkoa, en bonnes mains.

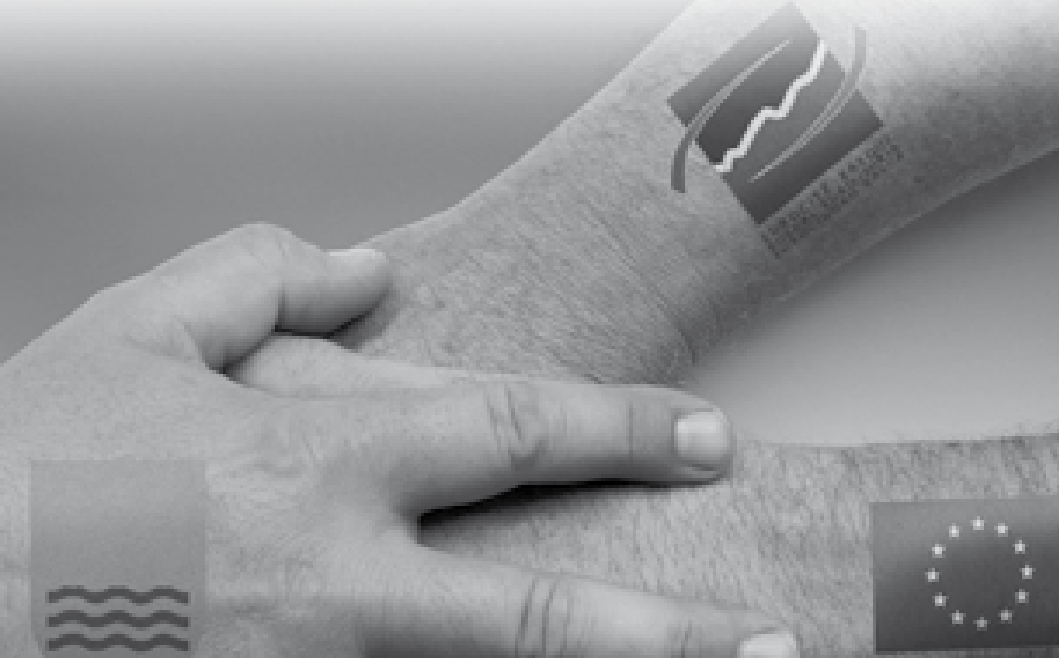

